\title{
Broadband Dual-Polarized Stacked Patch Antenna with High Isolation
}

\author{
Jia-yi REN ${ }^{1, *}$, Hu YANG², Xiang LIU ${ }^{3}$, Xian-bin LI ${ }^{4}$ and Jun-ru \\ GONG $^{5}$ \\ ${ }^{1 *},{ }^{2}$ National University of Defense Technology, Changsha, Hunan province, China \\ ${ }^{3}$ Hunan Institute of Science and Technology, Yueyang, Hunan province, China \\ ${ }^{4} \mathrm{Naval}$ Academy of Armament, Beijing, China
}

${ }^{5}$ Southwest Electronic and Telecommunication Technology Research Institute, Chengdu, Sichuan province, China

Keywords: Patch antenna, H-shaped slot, Wideband, High isolation.

\begin{abstract}
In this paper, a wideband dual-polarized stacked patch antenna at S-band with high port isolation is presented. By adopting the structure of $\mathrm{H}$-shaped coupling slots placed in " $T$ " configuration, the proposed antenna achieves dual polarizations and high port isolation, and the impedance bandwidth is significantly enhanced by the use of stacked structure. The antenna is designed, fabricated and measured. The measured return loss exhibits an impedance bandwidth $(\mathrm{S} 11<-10 \mathrm{~dB})$ of over $31 \%$ at both ports. The isolation between two ports is better than $40 \mathrm{~dB}$ and cross-polarization discriminations in both $\mathrm{E}$ and $\mathrm{H}$ planes are better than $21 \mathrm{~dB}$ within the forward half-space over the entire band.
\end{abstract}

\section{Introduction}

Dual-polarized antennas have been widely used in modern wireless communication systems for their ability to mitigate the multipath fading problem and increase channel capacity by polarization diversity. Microstrip antennas are an attractive option due to their advantages, such as low profile, low cost and being easy to fabricate. To meet the dual-polarized system requirements on port isolation, cross-polarization level and bandwidth, various kinds of dual-polarized patch antennas have been developed [1]. As the isolation between two orthogonal polarization ports plays a vital role, substantial efforts have been made to improve it. The methods could be using slot-coupled feed [2], hybrid feeds [3], dual-feed for single port [4], dual capacitive feeds with open-circuited microstrip stubs [5] or slot loaded technique [6] and so on.

In this paper, a dual-polarized stacked microstrip patch antenna using $\mathrm{H}$-shaped coupling slots in " $T$ " configuration is presented. The proposed antenna achieves high port isolation and low cross-polarization level. A parametric analysis of the impendence matching and isolation is performed. Experimental result shows good agreement with simulation. Both theoretical and experimental results are presented and discussed.

\section{Antenna Design and Parametric Analysis}

The configuration of the proposed dual-polarized slot-coupled antenna is shown in Fig. 1. The antenna consists of three dielectric substrate layers 1,2 , and 3, and one air layer. The upper circular patch is printed on the back of substrate 1 . The lower circular patch is printed on substrate 2. An air layer separates the two patches, for the purpose of 
extending the impedance bandwidth. The microstrip feed lines are put on the back of substrate 3, while ground plane with two slots is inserted between substrates 2 and 3 . The slots are in H-shape, and arranged in " $T$ " configuration to enhance the coupling between the feed line and the patch, and to improve the isolation between two ports. Power from different ports gets coupled to the radiating patch through the slots in the ground plane, producing linear polarization respectively.

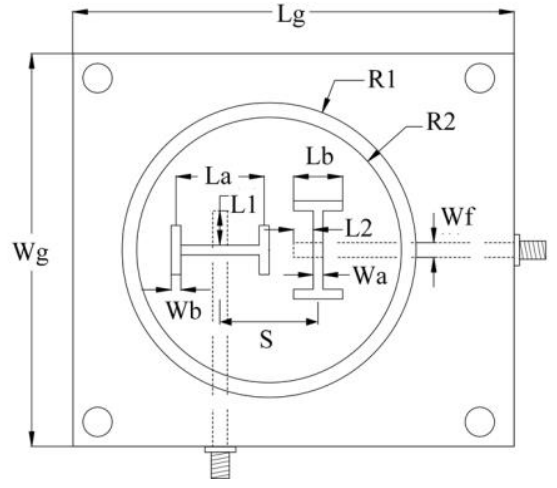

(a)

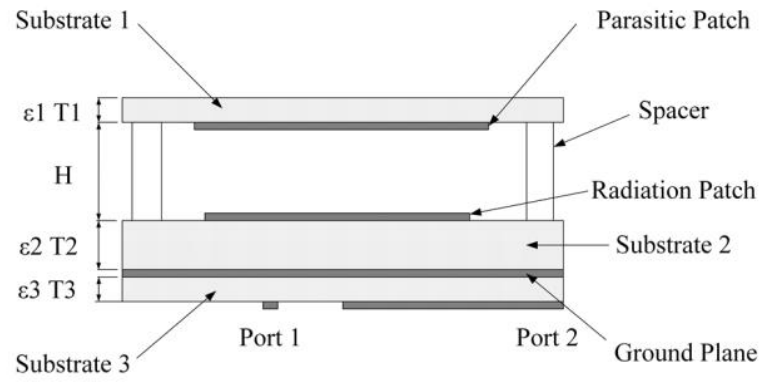

(b)

Figure 1. Geometry of the proposed antenna (a) Top view (b) front view

The antenna design is achieved by tuning the sizes of the patches, the open-stub lengths, the slot dimensions, the ground-plane dimensions and the air gap thickness. The effects of the vital parameters on impendence matching and isolation are studied. The influence of patches size is omitted here. During the parametric study, only one of these parameters in the aforementioned is changed, while other parameters are fixed. Fig. 2 shows the reflection coefficient of port 1 as a function of vital parameters. The slot dimensions $W a, W b, L a, L b$, influence the coupling between the feed line and the patch, which lead to affection on the antenna impendence. We can see from the Fig. 2-(a) (d), the antenna gets good coupling with certain dimensions, the change of the slot dimensions could make the impendence matching getting worse, and they mainly affect the lower resonant frequency. The open-stub lengths of the feed line beyond the center of the slot $L 1, L 2$, is used to tune excess reactance of the coupled antenna. As can be seen from Fig. 2-(e), the stub lengths affect the impendence matching over the whole band. In addition, the air gap thickness $H$ influences the coupling between the patches, which is an important part in the complete antenna impendence. As shown in Fig. 2-(f), the air gap thickness mainly affects the higher resonant frequency. As for the port isolation, the ground-plane size and the distance between two slots $S$, are extremely important influence factors. The isolation is significantly affected due to diffraction at the edges of the finite ground plane [7]. As shown in Fig. 3-(a), the mutual coupling between the two ports decreases obviously when the square ground-plane length increases. It is also concluded that the isolation is not improved much after a particular ground-plane size. The Fig. 3-(b) shows the isolation is slightly reduced with the decrease of the spacing between two slot centers.

Eventually, an antenna with center frequency $2.6 \mathrm{GHz}$ is designed using full-wave simulation software HFSS 15. The final parameters after optimization are listed in Table 1. The simulated return loss shows a bandwidth of $33.8 \%$ from 2.21 to $3.11 \mathrm{GHz}$ $(\mathrm{S} 11<-10 \mathrm{~dB})$ at port 1 and a bandwidth of $30.7 \%$ from 2.26 to $3.08 \mathrm{GHz}$ at port 2 . The simulated isolation between the two ports achieves over $40 \mathrm{~dB}$ across the entire band. 


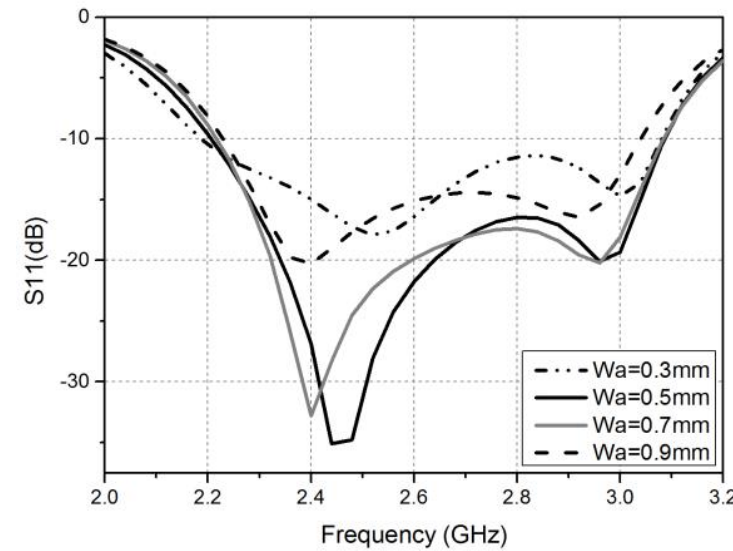

(a)

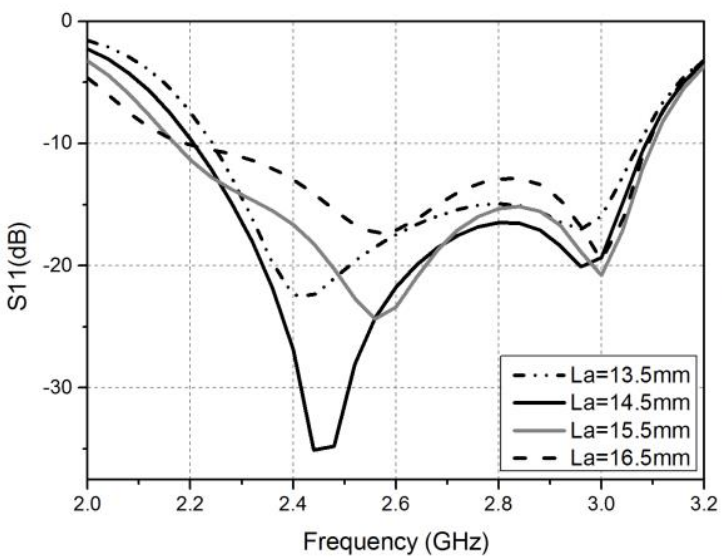

(c)

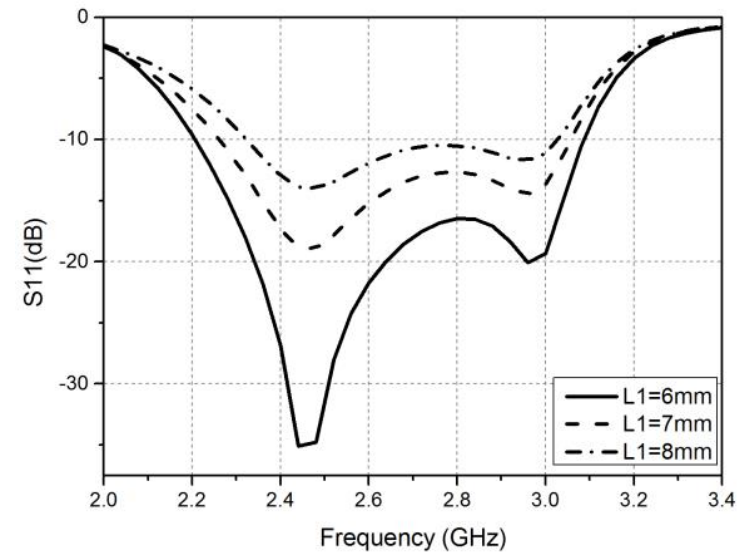

(e)

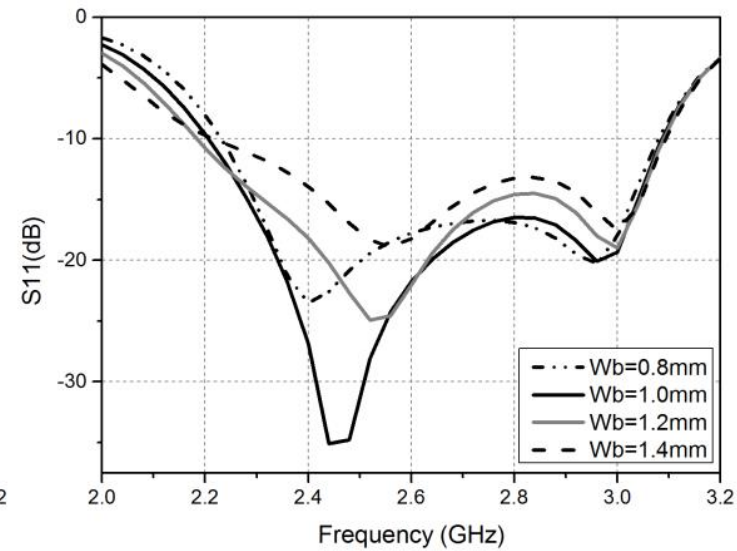

(b)

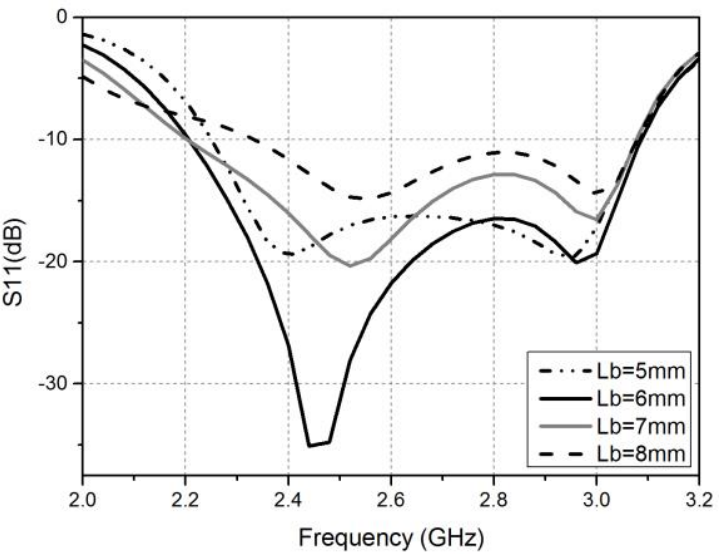

(d)

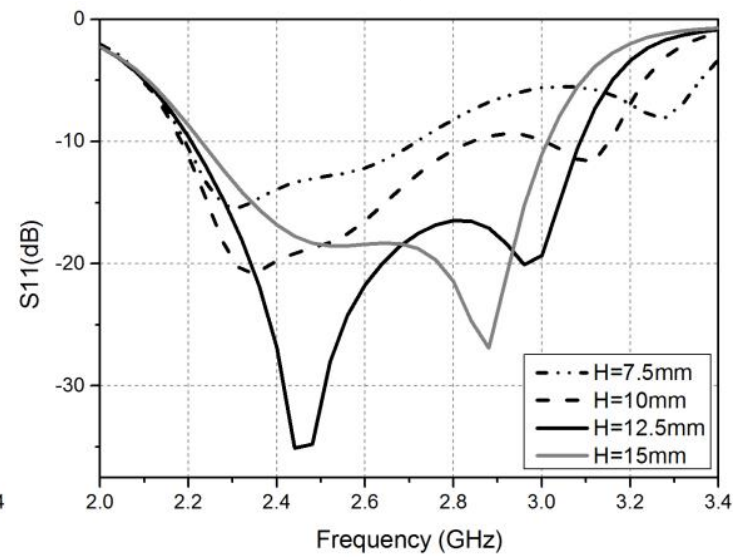

(f)

Figure 2. Effects on the reflection coeffiency at single port of different parameters 


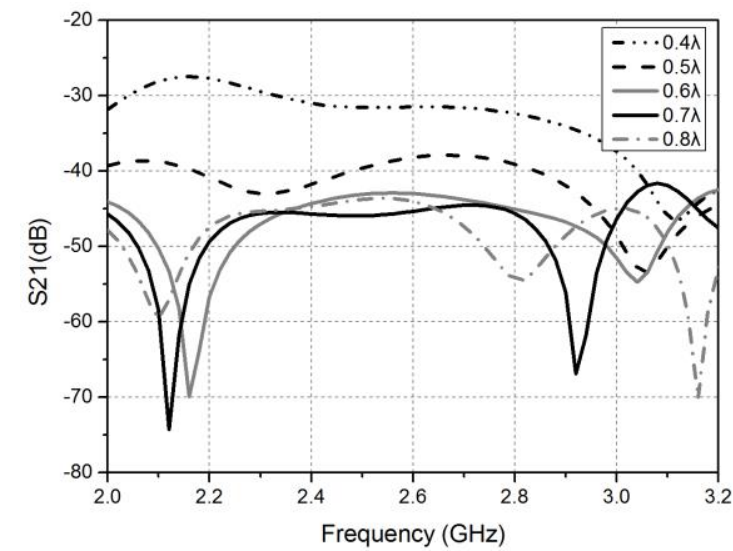

(a)

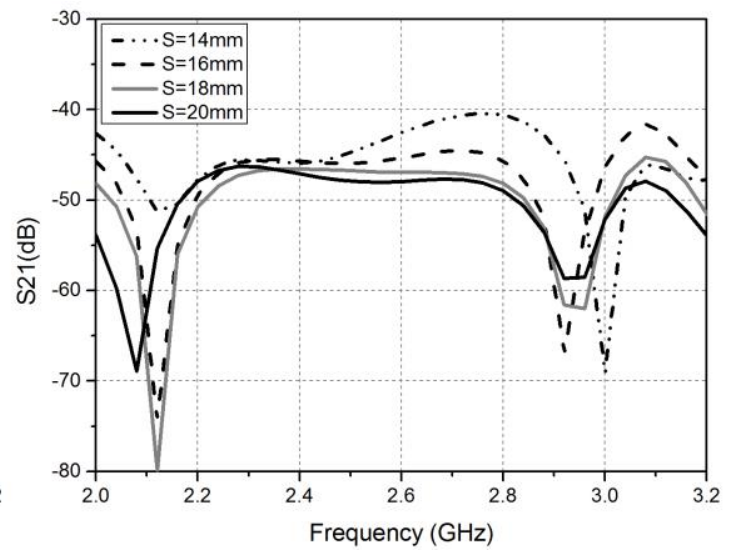

(b)

Figure 3. Effects of the (a) ground-plane size and (b) slots distance on the mutual coupling between ports

Table 1. Parameters of the designed antenna

\begin{tabular}{|c|c||c|c||c|c|}
\hline Parameter & Value $[\mathrm{mm}]$ & Parameter & Value $[\mathrm{mm}]$ & Parameter & Value $[\mathrm{mm}]$ \\
\hline$L g$ & 88 & $L a$ & 14.5 & $T 1$ & 1.0 \\
\hline$W g$ & 80 & $W a$ & 0.5 & $T 2$ & 5.0 \\
\hline$W f$ & 1.2 & $L b$ & 6 & $H$ & 12.5 \\
\hline$R l$ & 21 & $W b$ & 1 & $T 3$ & 0.8 \\
\hline$R 2$ & 20 & $L 1$ & 6 & $\varepsilon l, \varepsilon 2$ & 2.2 \\
\hline$S$ & 16 & $L 2$ & 5.7 & $\varepsilon 3$ & 6.0 \\
\hline
\end{tabular}
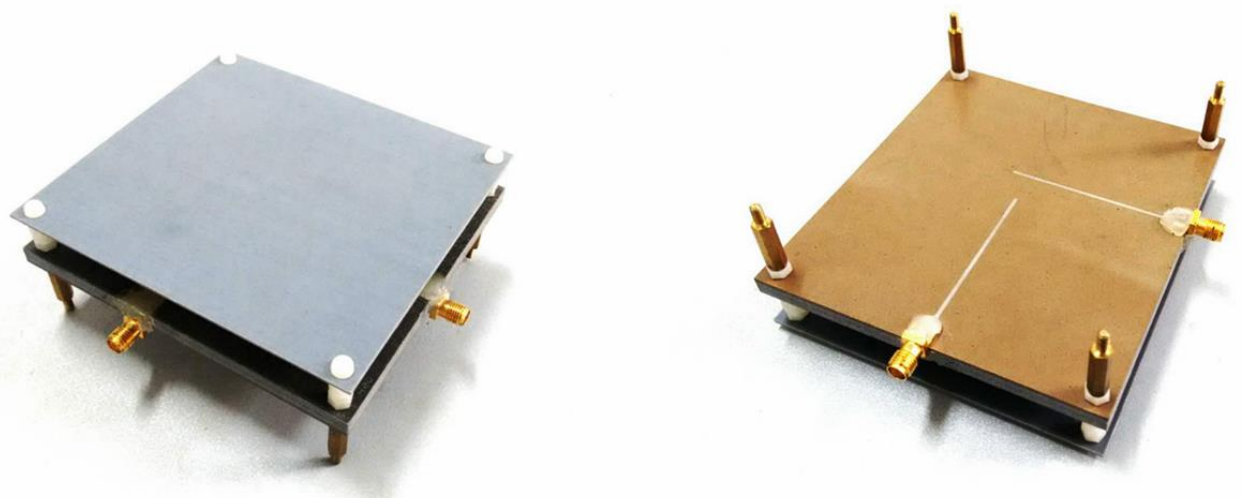

Figure 4. Photos of the fabricated antenna

\section{Experimental Results and Discussion}

The fabricated antenna is shown in Fig. 4. The measured and simulated results at different ports are depicted in Fig. 5. It can be seen that port 1 achieves 10-dB return loss bandwidth of 2.25-3.10 GHz, and port 2 achieves bandwidth of $2.25-3.08 \mathrm{GHz}$, the isolation of the antenna is above $40 \mathrm{~dB}$ over the whole band. 


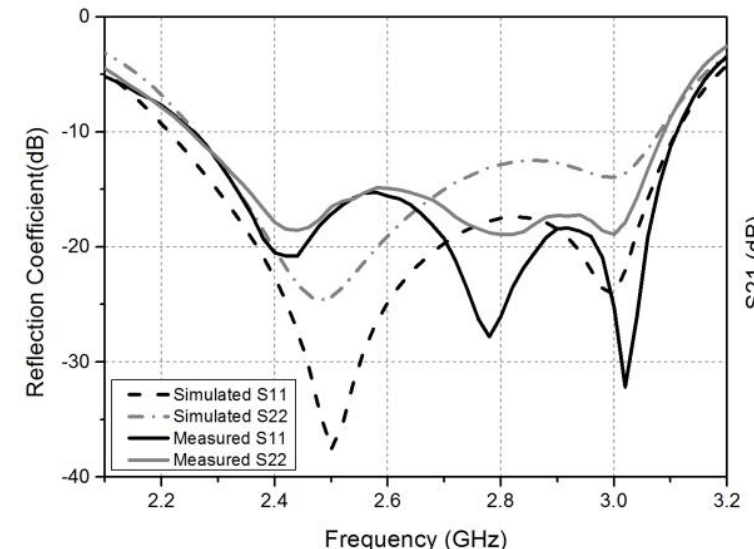

(a)

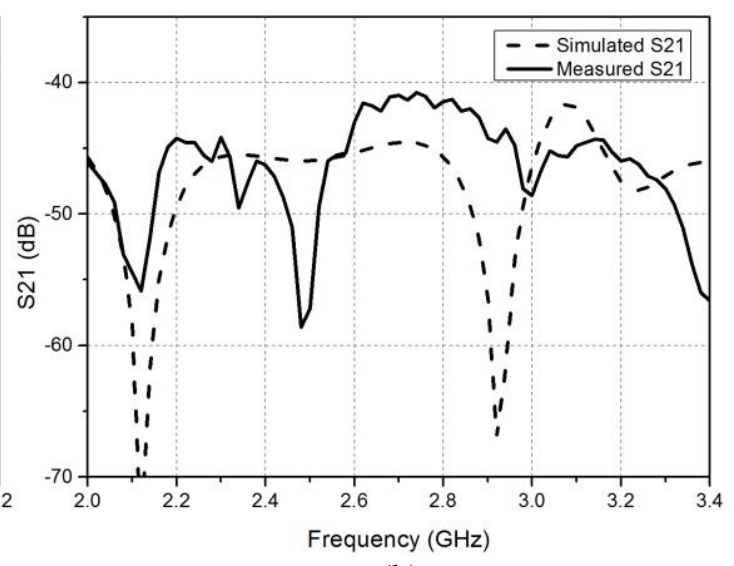

(b)

Figure 5. Measured and simulated S parameters of the antenna

Fig. 6 shows the measured and simulated normalized radiation patterns for E-plane and H-plane at $2.6 \mathrm{GHz}$. Due to the symmetrical characteristic of the antenna, the radiation patterns of the two ports are essentially identical. The measured gain and cross-polarization discrimination in the forward half-space are shown in Fig. 7. It can be seen that the measured gain is slightly lower than simulation, and the measured cross polarization discriminations are more than $21 \mathrm{~dB}$ over the entire forward half-space across the whole band.

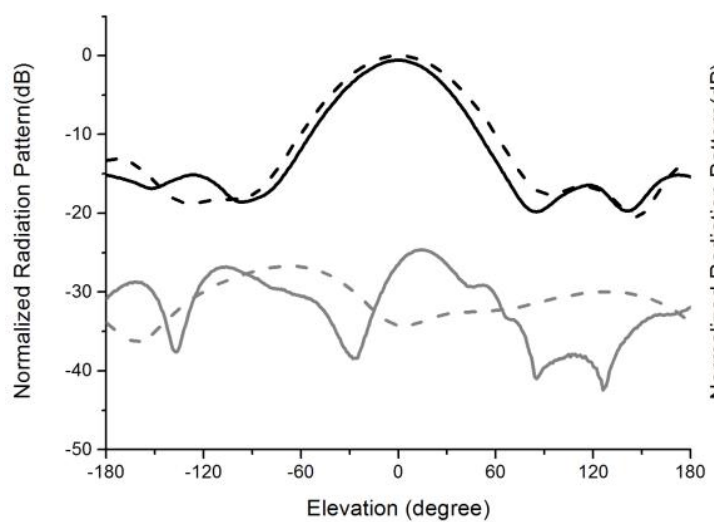

(a)

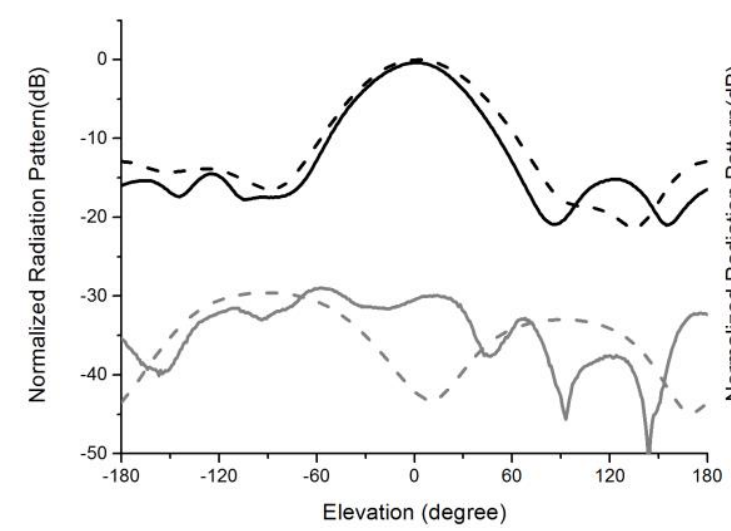

(c)

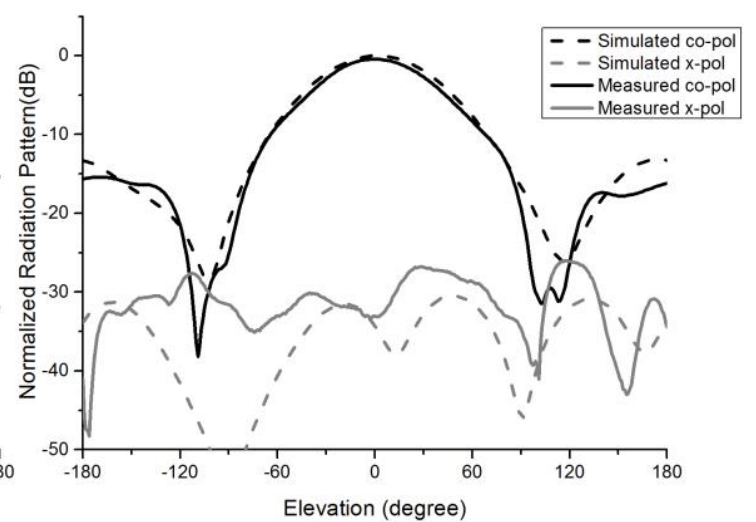

(b)

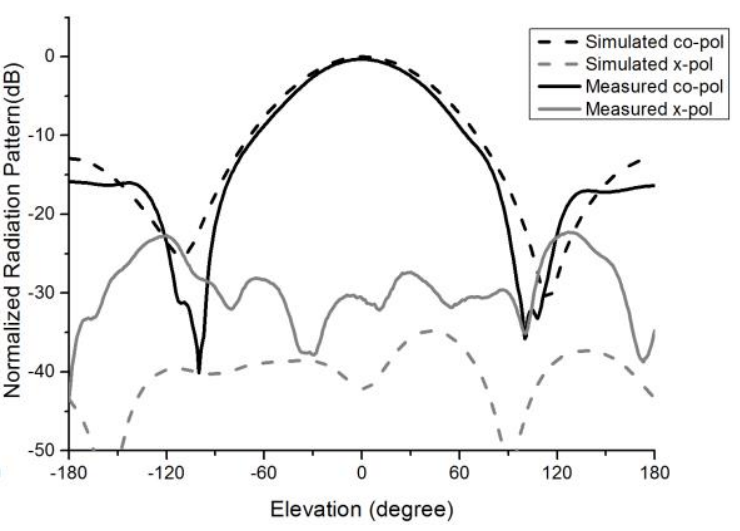

(d)

Figure 6. Measured and simulated normalized radiation patterns at $2.6 \mathrm{GHz}$. (a) E plane fed by Port 1

(b) H plane fed by Port 1 (c) E plane fed by Port 2 (a) H plane fed by Port 2 


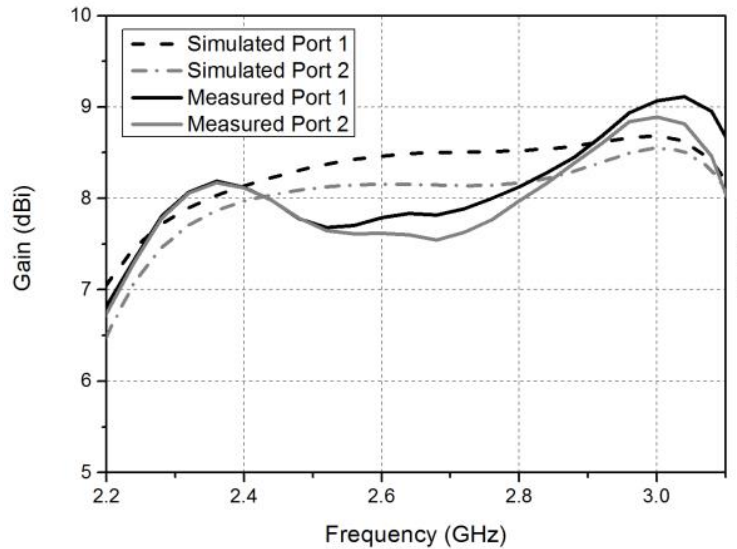

(a)

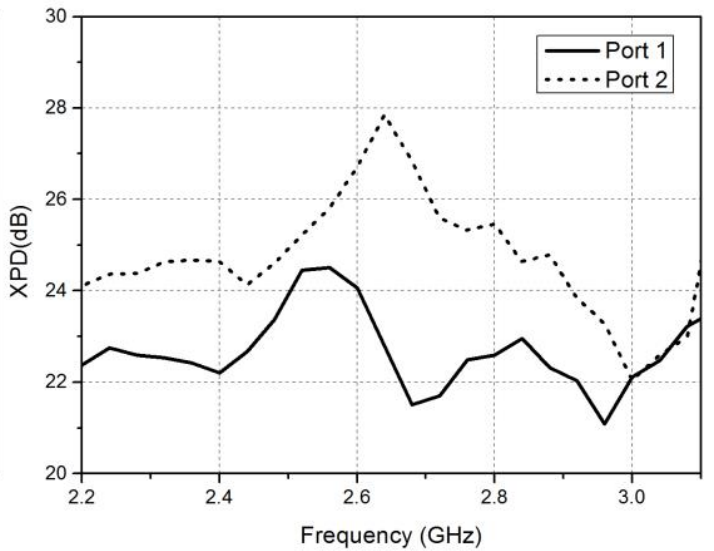

(b)

Figure 7. Measured gain and cross polarization discrimination (XPD) within the forward half-space

\section{Conclusion}

A wideband dual-polarized stacked patch antenna with high isolation and low cross polarization is designed. Compared with the existing patch antenna designs using $\mathrm{H}$-shaped slots, the port isolation of the proposed antenna is enhanced to $40 \mathrm{~dB}$, meanwhile the antenna achieves $21 \mathrm{~dB}$ high cross-polarization discrimination for both polarization across the whole band. The compact structure with broad-band, high isolation and low cross-polarization levels proves it promising for practical applications in various wireless systems.

\section{References}

[1] Mishra P K, Jahagirdar D R, Kumar G. A Review of Broadband Dual Linearly Polarized Microstrip Antenna Designs with High Isolation [Education Column][J]. IEEE Antennas and Propagation Magazine, 2014, 56(6): 238-251.

[2] Gao S C, Li L W, Leong M S, et al. Dual-polarized slot-coupled planar antenna with wide bandwidth[J]. IEEE Transactions on Antennas and Propagation, 2003, 51(3): 441-448.

[3] Chang C C, Row J S. Dual-feed dual-polarized patch antenna with low cross polarization and high isolation[J]. IEEE Transactions on Antennas and Propagation, 2009, 57(10): 3321-3324.

[4] Xie J J, Yin Y Z, Wang J H, et al. Wideband dual-polarised electromagnetic-fed patch antenna with high isolation and low cross-polarisation[J]. Electronics Letters, 2013, 49(3): 171-173.

[5] Son H W. Design of dual-polarised microstrip antenna with high isolation using capacitive feeds[J]. Electronics Letters, 2009, 45(11): 1.

[6] Zaman A U, Manholm L, Derneryd A. Dual polarised microstrip patch antenna with high port isolation[J]. Electronics Letters, 2007, 43(10): 551-552.

[7] Wong K L, Chiou T W. Finite ground plane effects on broad-band dual polarized patch antenna properties[J]. IEEE Transactions on Antennas \& Propagation, 2003, 51(4):903-904. 